\title{
ESTRESSE E ANSIEDADE EM GATOS DOMÉSTICOS: TRATAMENTO FARMACOLÓGICO E ETNOVETERINÁRIO - UMA REVISÃO
}

\author{
SILVA, Rafaela Pelisoli da ${ }^{1}$; \\ SUYENAGA, Edna Sayuri ${ }^{2}$.
}

Recebido: $19 / 12 / 2018$

Aceito: 03/04/2019

${ }^{1}$ Discente do Curso de Farmácia, Escola de Saúde, Universidade do Vale do Rio dos Sinos - UNISINOS; ${ }^{2}$ Docente Adjunta do Curso de Farmácia, Escola de Saúde, Universidade do Vale do Rio dos Sinos - UNISINOS.

\section{RESUMO}

$\mathrm{O}$ presente estudo visa discorrer sobre alternativas terapêuticas para gatos estressados e ansiosos através de uma revisão bibliográfica. Nesta busca, verificouse que a farmacoterapia designada ao público felino ainda é incipiente. A escassez de estudos pode ocasionar diagnósticos equivocados e, terapias indicadas a cães, quando aplicadas aos gatos, muitas vezes são inadequadas. Observou-se que dentre os medicamentos alopáticos prescritos, estão os antidepressivos tricíclicos e os inibidores seletivos de serotonina, os quais devem ser constantemente monitorados, pois podem ocasionar potenciais efeitos adversos. Há também, a possibilidade de uso de feromonas sintéticas na terapia comportamental ansiolítica. Dentre as espécies vegetais empregadas na etnoveterinária, destaca-se a Nepeta cataria, conhecida popularmente como erva-de-gato (catnip). Provavelmente, o seu efeito deva-se à ação de seu principal componente, a nepetalactona, sobre o sistema límbico do animal. Ao realizar uma análise in chemico, observa-se semelhança deste composto ao neurotransmissor serotonina, o que poderia justificar o bem-estar dos gatos, porém não há estudos farmacológicos e toxicológicos devidamente validados. Assim, ressalta-se a importância da orientação do uso racional de medicamentos e plantas medicinais, bem como o uso de métodos de enriquecimento ambiental para a redução do estresse e ansiedade em gatos domésticos, proporcionandoIhes maior qualidade de vida.

Palavras-chave: Comportamento felino. Ansiedade. Farmacoterapia. Etnoveterinária. 


\section{INTRODUÇÃO}

Historicamente, o homem apresenta afinidade com os animais no que tange a convivência, interação e domesticação. Tais hábitos estabeleceram um dos maiores e mais expressivos acontecimentos da humanidade, ainda que desconsiderando o nível de civilização atingido, animais estão inseridos nas culturas de todos os povos (LAMPERT, 2014). O crescente convívio entre animais e humanos, sobretudo cães e gatos, exige da medicina veterinária e dos profissionais atuantes, avaliações criteriosas baseadas em parâmetros mais bem estabelecidos no que se refere ao bem-estar e saúde do animal exposto a situações de ansiedade (FERREIRA et al., 2016). Segundo Bradshaw (2016), gatos domésticos, especialmente os que vivem em meio urbano, estão submetidos ao estresse crônico e assim, aos distúrbios de ansiedade. Levine (2008) reitera e elucida casos em que o animal expressa comportamentos agressivos em condições que não é capaz de evitar a circunstância causadora do medo.

No sentido de agregar conhecimento e auxiliar na condução terapêutica de gatos domésticos que apresentam sinais de estresse e ansiedade, a atual revisão descreve opções de tratamento disponíveis ressaltando seus efeitos adversos e possíveis implicações na qualidade de vida do animal.

\section{METODOLOGIA}

Para desenvolver a pesquisa foram utilizados sistemas eletrônicos de banco de dados como: ScienceDirect, Lilacs, Capes, Pubmed, Medline, Scielo, consulta em livros, revistas especializadas, teses, entre outros. As palavras-chave (em português e inglês) foram: comportamento felino, ansiedade, tratamento farmacológico, efeitos adversos, etnoveterinária, Nepeta cataria. Foram inclusos todos os títulos e resumos acerca do tema e exclusos trabalhos não relevantes ao assunto e publicados anteriores ao ano de 2000 , exceto estudos que apresentaram informações pontuais. 


\section{O ESTRESSE EM GATOS}

Há alguns anos a medicina realiza estudos que destacam e comprovam a influência do estresse sobre a saúde humana. No âmbito da medicina felina, o estresse é admitido como fator determinante da qualidade de vida e saúde dos gatos (MILLS et al., 2014).

O conceito de bem-estar animal sob a perspectiva da medicina veterinária baseia-se no fornecimento adequado de alimento e água fresca, acesso a um ambiente limpo com abrigo, temperatura, luz e barulho apropriados, cuidados veterinários quando necessário (ex.: vacinação, esterilização, desparasitação), liberdade para expressar a maioria dos comportamentos instintivos da espécie, face ao ser humano ou animais da mesma espécie, e proteção frente as ocasiões desencadeadores de medo e ansiedade (BROOM; MOLENTO, 2004). Para classificar o bem-estar animal deve-se avaliar as características próprias do indivíduo e compreender que elas são passíveis de mudanças (BROOM, 1986).

As razões para o surgimento do estresse em gatos domésticos podem estar atreladas a diversas alterações, tais como: mudanças ambientais (ex.: lugares e objetos), variações climáticas, modificação na dieta, ruídos altos ou desconhecidos, manejo das caixas de areia e cama, sobretudo quando exposto a novos humanos, outros gatos ou animais em seu território pessoal (STELLA et al., 2013). Os fatores causadores de estresse supracitados devem ser analisados sob uma ótica apurada, considerando essas fontes, por vezes, subjetivas. É importante salientar a atenção especial que se deve atribuir aos gatos que dividem habitat com outros animais, pois estudos revelam que dentre os diversos gatilhos desencadeadores de estresse, a disputa territorial é o mais expressivo (CAMERON et al., 2004).

Há autores que justificam os transtornos comportamentais dos gatos de estimação à prática mais comum, utilizada para auxiliar na domesticação, a clausura. Outros, reconhecem a carência de estudos abordando as necessidades do felino, da mesma forma que admitem a escassez de estímulo proporcionado pelo meio em que o gato está inserido, a ausência ou lacuna na relação entre gato e tutor, além da dificuldade em compreender e propagar conhecimentos fidedignos acerca do comportamento felino (GAZZANO et al., 2015). 
O estresse é compreendido como o conjunto de reações cognitivas, emocionais e somáticas a diversos estímulos (agradáveis ou repulsivos). No contexto fisiológico, desempenha o papel de preservar a estabilidade física e psicológica do animal. Logo, o estresse não se manifesta somente em episódios adversos, mas em quaisquer circunstâncias que ocorra desequilíbrio na homeostasia animal, considerando-o reflexo natural e saudável, desde que disparado pontualmente (LEVINE, 2008).

Este resultado fisiológico, denominado estresse, é acionado através das emoções, tais como medo e ansiedade, e irão possibilitar que o animal corresponda aos estímulos de maneira apropriada. Enquanto o medo promove uma reação adaptativa da parte animal, evitando condições de risco iminente; a ansiedade - por vezes, expressa através de comportamento agressivo - é decorrência da incapacidade felina de impedir o evento desencadeador de medo (LEVINE, 2008).

Apesar do estresse ser indicativo essencial e saudável em episódios pontuais, manifestações frequentes podem contribuir para que estados físicos e emocionais patológicos sejam desencadeados. Para estimar se o estresse que acomete o gato é agudo ou transitório, recomenda-se avaliação dos índices fisiológicos, a citar o cortisol, enquanto para o estresse crônico, é importante considerar as variações fisiológicas, sobretudo as comportamentais (IKI et al., 2011).

O estresse, o medo e a ansiedade atuam sobre estruturas semelhantes do sistema nervoso central (SNC), sendo elas o tálamo, o hipotálamo e a amígdala, preparando o animal, aumentando a atividade cortical e o estado de alerta, permitindo uma rápida resposta defensiva (LEVINE, 2008).

A frequente estimulação do sistema nervoso simpático, dispara uma série de reações químicas que reduzem as respostas imunológicas, sobretudo quando ocorrem por longos períodos, gerando um estresse de caráter crônico (LEVINE, 2008). Podendo, ainda, causar uma desordem nas funções hormonais das glândulas adrenais (LITTLE, 2011). 


\section{O PERFIL DO GATO ESTRESSADO}

O gato é demasiado vulnerável a mudanças repentinas e a partir disso é capaz de desenvolver um quadro de estresse que poderá ser representado através da redução da ingesta de alimentos e água, dejeção fora da caixa de areia ou ausência de eliminação de fezes, extrapolando, eventualmente, 24 horas, elevando a possibilidade de constipação. Geralmente quando a razão do estresse está atrelada ao isolamento ou clausura, é comum o gato apresentar episódios de diarreia e vômito intermitente, além da redução do apetite (MILLS et al., 2014).

Como mencionado anteriormente, o sistema imune é capaz de combater patógenos mesmo frente a determinadas situações desencadeadoras de estresse, todavia quando o estresse é classificado como crônico, a resposta pode ser oposta. Gatos acometidos pelo estresse crônico são altamente suscetíveis às doenças respiratórias. Também é comum patógenos em estados subclínicos se manifestarem - a citar o herpes vírus felino tipo I - quando ocorre a coibição da produção de anticorpos encontrados na mucosa, especificamente IgA, acentuando a vulnerabilidade às infecções respiratórias (GOURKOW et al., 2014).

Além disso, os gatos podem apresentar o hábito de lamber o próprio pelo ou arrancá-lo em virtude dos gatilhos indutores de estresse, frustração, ansiedade ou devido simplesmente a uma doença dermatológica (WAISGLASS et al., 2006). Apesar do costume de lamber o próprio pelo, também denominado "autogrooming", ser comum e exercer papel fundamental para manutenção saudável da pele e as características do pelo animal, pode ser indício sugestivo de estresse, seja no âmbito social como não social (IKI et al., 2011).

Segundo Mills et al. (2014), o felino acometido pelo estresse no período da gestação dispõe de um sistema imunológico frágil e concederá o mesmo à prole. A dificuldade que o gato possui de enfrentar estímulos desconhecidos torna-o propenso a produzir pouco leite, reduzir a prole sobrevivente, diminuir a taxa de crescimento e por consequência elevar o índice de mortalidade e o número de descendentes (BROOM, 1986). 


\section{TRATAMENTO FARMACOLÓGICO}

Os tratamentos farmacológicos usualmente utilizados em casos de comportamento atípico por parte do animal podem ser necessários somente por um breve período, até que a conduta anormal seja cessada ou o fator estressor seja extinto. Os fármacos prescritos agem sobre os neurotransmissores, proporcionando efeito sedativo, antidepressivo ou ansiolítico. As classes de medicamentos mais utilizadas se dividem em antidepressivos tricíclicos (ATC), inibidores seletivos da recaptação da serotonina (ISRS) e os tranquilizantes benzodiazepínicos (SCOTT et al., 2001).

A razão de valer-se desses medicamentos baseia-se em atenuar os níveis de excitação, diminuir os estados ansiosos e controlar os impulsos. A partir do início do tratamento, o gato tende a estabelecer uma condição propensa às mudanças comportamentais (LEVINE, 2008). Entretanto, grande parte dos profissionais veterinários baseia-se na terapêutica humana ao prescrever e administrar esses medicamentos, visto que são poucas as substâncias comprovadas e registradas para uso animal (SIMPSON; PAPICH, 2003). É importante ressaltar que o efeito provocado pelos psicofármacos em humanos não corresponde obrigatoriamente ao mesmo efeito exercido sobre o animal, considerando que para alguns princípios ativos não há ensaios clínicos realizados em animais domésticos, exceto animais de laboratório ou mesmo em humanos (CROWELL-DAVIS; MURRAY, 2005).

Os antidepressivos tricíclicos (ATC) são fármacos amplamente utilizados para tratar desvios comportamentais em gatos, por exemplo: amitriptilina, clomipramina e doxepina. Essa classe atua inibindo a recaptação de noradrenalina e serotonina pelas terminações nervosas e bloqueando os receptores de glutamato e canais de sódio (ANDRADE, 2002). O tratamento terapêutico com tal classe de fármacos não é recomendado em casos agudos, pois esses fármacos apresentam efeito clínico tardio e devem ser administradas durante 30 dias, no mínimo. Ao passo que a retirada desses medicamentos deve ser feita gradativamente (ALHO, 2012). Ainda, podem ser observados alguns efeitos colaterais como sonolência, aumento de peso e até mesmo redução do "grooming" (CHEW et al., 1998). Apresentam como principais efeitos adversos o aumento da frequência cardíaca, midríase, redução da 
produção de lágrimas, retenção urinária e constipação (ANDRADE, 2002). Por isso, seu uso deve ser feito somente quando necessário e sob orientação profissional.

Os fármacos inibidores seletivos da recaptação da serotonina (ISRS) agem de modo a inibir a recaptação pré-sináptica de serotonina e noradrenalina, como consequência elevam a concentração de serotonina e bloqueiam os receptores de acetilcolina, dopamina e noradrenalina. A fluoxetina, paroxetina, fluvoxamina, sertralina e citalopram são exemplos de fármacos dessa classe (LANDSBERG et al., 2004). Embora atuem preferencialmente sobre os receptores serotoninérgicos, os ISRS apresentam em suas estruturas químicas grupamentos fenoxila ou fenila, os quais permitem uma interação molecular junto aos receptores colinérgicos, adrenérgicos e histaminérgicos, promovendo assim, o surgimento de efeitos colaterais atrelados à ação antagonista (ANDRADE, 2002). O início de ação desses medicamentos oscila entre 2 a 8 semanas e pode estar atrelado aos efeitos colaterais tais como inibição da ingesta de alimentos e eventuais episódios de vômito (HART et al., 2005).

Os benzodiazepínicos são habitualmente empregados na medicina veterinária por conferir propriedades sedativa, hipnótica, anticonvulsivante, relaxante muscular, ansiolítica e cessar distúrbios comportamentais (SIMPSON; PAPICH, 2003). O diazepam é um exemplo desta classe, apresenta meia vida de 5,5 horas em gatos domésticos (PLUMB, 2002). No entanto, estudos indicam a possibilidade de ocorrer falência hepática em felinos quando administrado por via oral (BAGLEY, 2006; SIMPSON; PAPICH, 2003). Todavia, este fármaco é usualmente administrado via retal ou intravenosa em medicina veterinária, não sendo recomendada a aplicação por via intramuscular devido ao seu veículo oleoso (PLUMB, 2002). Apresentam alta lipofilia, que possibilita atravessar a barreira hematoencefálica e placentária, bem como atingir as glândulas mamárias, devendo, portanto, evitar-se o uso em gatas gestantes e lactantes (CROWELL-DAVIS; MURRAY, 2005; SPINOSA et al., 2008).

Objetivando reduzir os efeitos colaterais provocados pela terapia alopática, veterinários e tutores de animais buscam muitas vezes tratamentos alternativos (OZAKI; DUARTE, 2006).

\section{ETNOVETERINÁRIA}

A Etnoveterinária estuda as práticas populares e aplicação de plantas medicinais no tratamento ou prevenção das doenças que acometem os animais (ALMEIDA et al., 2006). Este termo foi utilizado primeiramente por McCorkle, na década de 80, e propõe estudar as 
plantas medicinais empregadas no controle e cuidado do bem-estar animal (CONFESSOR et al., 2009).

Existem múltiplos fatores que contribuem para a propagação e preservação das práticas etnoveterinárias, dentre as quais se destacam: os elevados custos dos serviços veterinários, a dificuldade de adquirir fármacos alopáticos e a crescente demanda por alimentação orgânica, auxiliando o desenvolvimento desta ciência, sobretudo com a utilização de medicamentos fitoterápicos (MONTEIRO et al., 2011). Haja vista a ampla biodiversidade e variedade cultural que compõem o Brasil, os conhecimentos e técnicas etnoveterinárias ainda são ínfimos, reflexo da insuficiência de levantamentos e informações acerca do tema. Esse déficit causa danos à área de fitoterapia veterinária, à documentação científica a respeito do uso de plantas aplicadas no tratamento das inúmeras patologias que acometem os animais, causando dificuldades à validação e a propagação desses métodos (MONTEIRO et al., 2011).

\section{FITOTERAPIA VETERINÁRIA}

A aplicabilidade da fitoterapia veterinária se dá da mesma forma que a fitoterapia humana, podendo se apresentar na forma de chás, compressas, óleos, extratos, produtos inalatórios e cremes. O que de fato distingue a fitoterapia veterinária da fitoterapia humana são as dosagens empregadas (VÉGAS, 2007).

A Organização Mundial da Saúde (OMS) reconhece a resposta biológica satisfatória atrelada ao tratamento fitoterápico aplicado tanto em animais quanto seres humanos, dado que salienta a necessidade e relevância do saber específico acerca das plantas medicinais e pondera o efeito dos princípios ativos e toxicidade (OMS, 2013). Deste modo, a fitoterapia veterinária requer o mesmo cuidado e atenção que a Agência Nacional de Vigilância Sanitária (ANVISA) destina aos fitoterápicos humanos em relação aos parâmetros de controle. Segundo Bruno et al. (2016) as normas atuais, para os fitoterápicos veterinários, são amplas e genéricas, as vezes contraditórias, desestimulando as empresas em investir nesse segmento de mercado, sugerindo, então, uma revisão desse marco regulatório, utilizando como referencial a experiência na área humana, complementando e ajustando às peculiaridades do mercado de produtos farmacêuticos veterinários. 
Uma das espécies vegetais muito utilizadas para reduzir o estresse e ansiedade em gatos é a Nepeta cataria. Popularmente conhecida como erva-de-gato, hortelã-dos-gatos ou simplesmente catnip, pertence à família Lamiaceae. É uma planta perene, herbácea, nativa da Europa e de algumas regiões da Ásia (USDA, 2018).

A N. cataria manifesta nos felinos um comportamento peculiar, muitas vezes humorístico, indicativo de prazer extremo. Inicialmente utilizada com finalidade recreativa, a planta pode ser adquirida em lojas de animais na forma de folhas secas picadas, pós, líquido e spray. Dentro de pequenos brinquedos ou em arranhadores, na intenção de divertir e estimular o relaxamento dos felinos, acarretando um comportamento excêntrico assinalado por relaxamento, prazer e vocalização (BEAVER, 2005).

A planta produz uma resposta indicativa de prazer extremo na maioria dos felinos e efeito alucinógeno em humanos - equivalente ao LSD, porém reduzido. A erva apresenta odor característico - semelhante à mistura de hortelã e grama cortada - em razão do seu óleo essencial, constituído majoritariamente por nepetalactona, uma lactona monoterpênica, substância química responsável por atrair os felinos (HATCH, 1972).

No presente trabalho, foi realizada uma análise in chemico (relação estrutura química versus atividade farmacológica) da nepetalactona (4,7-dimetil-5,6,7,7a-tetraidro-ciclopenta[c]piran1(4aH)-ona), substância majoritária do óleo essencial de catnip, comparando-a com o neurotransmissor serotonina, responsável pelo humor, ansiedade e depressão, como estratégia preditiva na avaliação dos possíveis efeitos da planta $N$. cataria em gatos domésticos.

Ao analisar a estrutura química da nepetalactona, esta possui peso molecular de $166,21 \mathrm{~g} /$ mol $\left(\mathrm{C}_{10} \mathrm{H}_{14} \mathrm{O}_{2}\right)$. Além de ser uma lactona monoterpênica, com características lipofílicas, sugere-se que possa atravessar a barreira hematoencefálica e atingir o sistema nervoso do felino. Não há evidências científicas, mas indica-se que este composto possa atuar sobre os receptores serotoninérgicos, conferindo a sensação de bem-estar ao animal, uma vez que através da análise in chemico, o núcleo tetraidro-ciclopentano da nepetalactona assemelhase ao anel indol da serotonina (Figura 1), podendo haver uma interação isostérica. 


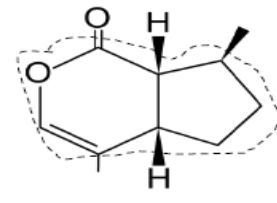

Nepetalactona

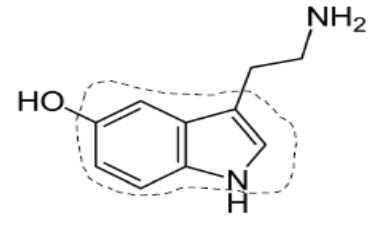

Serotonina

Figura 1 - Analogia estrutural entre a nepetalactona e a serotonina.

O papel da serotonina na ansiedade é pouco elucidado em gatos, ainda que considerado fundamental aos mamíferos (GRAEFF; HETEM, 2004). Este neurotransmissor atua no sistema nervoso central de múltiplas formas, podendo desempenhar ações inibitórias e/ou estimulatórias sobre as vias neuronais, o que dá suporte a algumas teorias que relacionam receptores serotoninérgicos aos fármacos ansiolíticos (BRAGA et al., 2010).

Estudos revelam que dois entre três gatos expostos ao catnip apresentam comportamentos que variam entre rolamento, esfregar o queixo e a bochecha, balançar a cabeça repetidamente, arranhar, lamber e farejar continuamente (BOL et al., 2017; TODD, 1963). O efeito é imediato e se estende por um breve período, de 5 a 15 minutos, aproximadamente, seguido de um estágio refratário por mais alguns instantes (TODD, 1963). Autores sugerem que todos os gatos expressam alguma resposta frente ao catnip, podendo classificá-la em ativa ou passiva, dependendo do comportamento expresso pelo felino após a exposição. A resposta ativa é caracterizada pela frequência de capotamento e grooming, enquanto a resposta passiva é determinada através, principalmente, da redução da atividade motora, diminuição da frequência de vocalizações e postura semelhante a esfinge por mais tempo (ESPÍN-ITURBE et al., 2017). Ainda, segundo Espín-Iturbe et al. (2017), fatores como idade, sexo e castração influenciam diretamente no comportamento do felino quando exposto ao catnip e consequentemente na resposta gerada.

Recomenda-se o uso comedido em fêmeas prenhes, já que possui capacidade de induzir o parto e, posteriormente, causar aborto (HATCH, 1972; OSTERHOUDT et al., 1997; SHERRY; HUNTER, 1979). A nepetalactona, princípio ativo da droga vegetal, demonstra possuir propriedades farmacológicas intrigantes sobre o sistema límbico de gatos (AYDIN et al., 1998; OSTERHOUDT et al., 1997). 
Zhu et al. (2009) pesquisaram a toxicidade do óleo essencial obtido das folhas de catnip e frente à administração oral, dérmica, inalatória, irritação primária da pele e olhos em roedores. De modo geral, o óleo essencial de catnip apresentou baixíssimos índices de toxicidade aguda. Contudo, ocorreu o desenvolvimento de eritema quatro dias após a aplicação tópica em todos os animais testados, o que propõe que o óleo essencial de catnip provoque irritação na pele. Em relação aos testes por inalação, não foram observados sinais clínicos apontando intoxicação, tampouco mortes ou irregularidades nos roedores de ambos os sexos, após o período de duas semanas. Quanto ao teste de irritação ocular, foram verificados sinais de irritação conjuntival após uma hora de exposição ao material de teste, que não persistiu por 24 horas. Não houve presença de nenhum outro sinal de toxicidade aguda durante o período de testes.

\section{OUTROS FITOTERÁPICOS}

As folhas do maracujá-vermelho (Passiflora incarnata) são utilizadas em situações de excitação constante e promovem o relaxamento e sedação no animal, devido a ação produzida por seus metabólitos secundários, os flavonoides e alcaloides (BARBOSA, 2011). Sugere-se $2 \mathrm{~mL}$ de tintura, três vezes ao dia, durante 90 dias ou $3 \mathrm{~mL}$ por 21 dias (CAVALCANTI, 1997).

O uso de infuso de camomila (Matricaria chamomilla) proporciona efeitos sedativos promovido pelo seu óleo essencial em condições de estresse (ABEBE, 2002; MANN; STABA, 1986).

O lúpulo (Humulus lupulus) é utilizado no tratamento de distúrbios do sistema nervoso em animais. Recomenda-se de 2 a $3 \mathrm{~mL}$ de tintura ao dia, durante 21 dias (CAVALCANTI, 1997).

Não foram relatadas as posologias para as seguintes espécies: Ageratum conyzoides L. utilizada no controle da ansiedade e insônia (CUNHA et al., 2012); Valeriana officinalis indicada como sedativo, relaxante muscular e indutor de sono (CUNHA et al., 2012) e folhas da Melissa officinalis, utilizadas no tratamento de estresse e ansiedade em pets (PEETERS et al., 2004). 


\section{FEROMONAS}

Segundo Overall (2013), o gato dispõe de uma mucosa nasal de aproximadamente $20 \mathrm{~cm}^{2}$, que possui 200 milhões de células receptoras - o dobro de dimensão do epitélio olfatório humano - que permite ao gato um olfato 30 vezes mais apurado quando comparado ao do homem (PEREIRA; PEREIRA, 2013). Ademais, os gatos utilizam os estímulos olfativos para analisar seu entorno e elevar sua sensação de segurança e conforto (ELLIS et al., 2010, 2013). O seu olfato além de ser mais sensível que o do ser humano, possui um aparato olfativo auxiliar denominado órgão vomeronasal, responsável pela detecção de feromonas (BRADSHAW, 2018), que por sua vez, são substâncias presentes em fluidos como: urina, suor, glândulas exócrinas e secreções genitais, liberadas em situações estressantes ou desconhecidas, com potencial de induzir alterações comportamentais, endócrinas e imunológicas dos animais receptores da mesma espécie (KRONEN et al., 2006). Este órgão vomeronasal, também denominado órgão de Jacobson, trata-se de uma estrutura epitelial tubular circundada por vasos sanguíneos, localizada na região final do palato duro e das narinas (PEREIRA; PEREIRA, 2013). Terminações nervosas que inervam o órgão em questão conectam o bulbo olfatório à amígdala vomeronasal, responsável pelo bem-estar do animal (VILANOVA, 2003).

Assim, em relação às feromonas faciais de felinos, foram isoladas cinco misturas funcionais da região perioral e bochecha (F1 a F5). Destas, são produzidas sinteticamente a F3 e F4 e estão disponibilizadas comercialmente em difusores plug-in ou através de spray. Estas feromonas sintéticas facilitam a percepção de familiaridade dos objetos aos gatos. Ambas as formulações parecem ser igualmente eficazes (MILLS, 2005). Sugere-se que estes compostos atuem sobre o sistema límbico e induzam a redução do cortisol e, consequentemente, ocorra uma modificação do estado emocional dos gatos, reduzindo o estresse, sobretudo em ambientes desconhecidos (SILVA et al., 2017). A possibilidade de falha com este tipo de tratamento pode ser devido a uma tendência de se tratar os sinais da ansiedade, sem estabelecer a natureza do problema, ou por uma intervenção comportamental inadequada (MILLS, 2005). 


\section{CONSIDERAÇÕES FINAIS}

Estudos sugerem que o gato doméstico, Felis silvestres catus, procedeu do gato selvagem africano, Felis silvestres libyca, e que desde os primórdios o convívio entre humanos e felinos estabeleceu-se em decorrência das aptidões para a caça a roedores que esses animais detêm (BEAVER, 2005). De acordo com Jongman (2007), os gatos apresentam habilidade de se adaptar relativamente bem a ambientes delimitados, sejam pequenas residências, apartamentos ou abrigos, o motivo pelo qual os tornaram populares como animais de companhia.

Alguns autores afirmam que o enriquecimento ambiental com o acréscimo de um ou mais fatores, contribuem para o aumento da complexidade de um ambiente relativamente empobrecido (HOLM; LADEWIG, 2007), e referem-se a métodos que estimulam as condições de bem-estar psicológico do animal somente por favorecer novas possibilidades comportamentais, que além de assegurar a integridade fisiológica e comportamental, atenuam comportamentos atípicos e desenvolvem habilidades frente a desafios e novas experiências em cativeiro (MASON et al., 2007). Porém, o isolamento ou clausura em ambientes empobrecidos, pode levar o gato a apresentar episódios de estresse e ansiedade (OLSSON et al., 2003).

Com o propósito de atenuar quadros de estresse e ansiedade, reduzir excitação, diminuir comportamentos característicos de compulsão, como movimentos repetitivos, que muitas vezes contribuem para a manutenção de desordens orgânicas, muitos profissionais veterinários norteiam-se pela terapêutica humana para indicar e administrar esses medicamentos, visto que são poucas as substâncias registradas e autorizadas para empregar em animais (SIMPSON; PAPICH, 2003).

Deve-se atentar ao fato de que psicofármacos não apresentam necessariamente efeitos similares quando administrados em humanos e animais de companhia. Posto que, para diversas substâncias, não foram realizados estudos clínicos em cães e gatos, exceto animais de laboratório (camundongos, cobaias, ratos) ou mesmo humanos. Não obstante, é dever do médico veterinário verificar se os tutores utilizam fármacos prescritos a humanos em seus animais, sem as devidas orientações. Desta forma, é imprescindível manter registros 
minuciosos da consulta, realizar prescrições precisas, ou seja, que a posologia corresponda ao tempo de tratamento, além de monitorar o paciente com regularidade (CROWELL-DAVIS; MURRAY, 2005).

Gatos jovens demonstram maior suscetibilidade a agentes tóxicos em virtude do metabolismo e vias de eliminação se apresentarem ainda imaturos. Em contra partida, felinos idosos podem manifestar reações adversas frente a algumas substâncias em razão do metabolismo ou vias de eliminação já terem sofrido algum dano (POPPENGA, 2002). Além disso, felinos apresentam deficiências ou limitações em algumas vias metabólicas, incluindo a glucuronidação hepática, hidroxilação e desmetilação. Quando comparado ao metabolismo de cães e humanos, os gatos usam diferentes isoenzimas para metabolizar fármacos (PLUMB, 2015).

Os produtos naturais têm demonstrado grande êxito e pode-se atribuir isto às constantes pesquisas realizadas principalmente no seguimento da Etnofarmacologia, em que se incluem as plantas medicinais (KINGHORN et al., 2011; NEWMAN et al., 2003).

Segundo Passos et al. (2009), muitos componentes presentes em óleos voláteis intensificam a neurotransmissão GABAérgica de camundongos, indicando o seu potencial para o desenvolvimento de fármacos ansiolíticos e/ou anticonvulsivantes.

Com relação à inalação de óleos voláteis com finalidade terapêutica realizada por gatos domésticos, é importante salientar a ausência de métodos validados para tal prática, bem como a mensuração de substância inalada. Tendo em vista também, a necessidade de padronizar a extração de óleos voláteis para garantir segurança ao animal exposto.

Não foram realizados estudos científicos em felinos quanto ao uso das espécies vegetais mentrasto, melissa, passiflora, camomila e ginseng como tratamento alternativo para ansiedade.

Desta forma, a validação dos efeitos farmacológicos de plantas empregadas na etnoveterinária se faz necessária, para uma farmacoterapia mais eficaz e segura. 


\section{CONCLUSÃO}

Há carência de pesquisas científicas validadas a respeito da atividade farmacológica e toxicológica de espécies vegetais sobre o sistema nervoso central de felinos, especialmente a catnip, não devendo utilizá-la de maneira indiscriminada pelo fato de ser um produto natural. Como medida não farmacológica, é recomendado o enriquecimento do ambiente, podendo ser associado à inclusão de feromonas como terapia comportamental, para redução do nível de estresse e ansiedade dos felinos. Em casos específicos, faz-se necessário o uso de antidepressivos, porém com cautela e acompanhamento médico assistido, devido aos potenciais efeitos adversos.

É importante que o profissional veterinário e o farmacêutico trabalhem de forma integrada, a fim de conceder suporte técnico-científico na utilização de medicamentos e plantas medicinais, indicando produtos que de fato tenham sido testados e aprovados e orientando os tutores sobre o seu uso racional. Desta forma, contribui-se para a promoção da saúde dos animais de companhia, proporcionando-lhes melhor qualidade de vida.

\section{STRESS AND ANXIETY IN DOMESTIC CATS: PHARMACOLOGICAL AND ETHNOVETERINARY TREATMENT - A REVIEW}

\section{ABSTRACT}

T he present study aims to discuss therapeutic alternatives for stressed and anxious cats through a bibliographic review. In this search, it was verified that the pharmacotherapy designated to felines is still incipient. The scarcity of studies may lead to misdiagnosis and therapies indicated to canines are often inadequate to felines. It has been observed that among the allopathic drugs commonly prescribed are tricyclic antidepressants and selective serotonin inhibitors, which should be constantly monitored as they may cause potential adverse effects. There is also the possibility of using synthetic pheromones in anxiolytic behavioral therapy. Among the vegetable species used in ethnoveterinary, Nepeta cataria is emphasized, popularly known as catnip. Probably, its effect is due to the action of its main component, nepetalactone on the limbic system of the animal. When performing an in chemico analysis, a similarity of this compound to the neurotransmitter serotonin is observed, which could justify the well-being of the cats, but there are no validated pharmacological and toxicological studies. Thus, the importance of 
the orientation for the rational use of drugs and medicinal plants, as well as the use of environmental enrichment methods for the reduction of stress and anxiety in domestic cats is emphasized, providing quality of life for the animals.

Keywords: Feline behaviour. Anxiety. Pharmacotherapy. Ethnoveterinary.

\title{
ESTRÉS Y ANSIEDAD EN GATOS DOMÉSTICOS: TRATAMIENTO FARMACOLÓGICO Y ETNOVETERINARIO - UNA REVISIÓN
}

\section{RESUMEN}

E

presente estudio trata de disuadir alternativas terapéuticas para gatos estresados y ansiosos a través de una revisión bibliográfica. En esta búsqueda, se verificó que la farmacoterapia designada al público felino aún es incipiente. La escasez de estudios puede ocasionar en diagnósticos equivocados. Además, terapias indicadas a perros, cuando se aplican a los gatos, a menudo son inadecuadas. Se observó qué entre los medicamentos alopáticos prescritos, están los antidepresivos tricíclicos y los inhibidores selectivos de serotonina, los cuales deben ser constantemente monitoreados, pues pueden ocasionar potenciales efectos adversos. Hay también, la posibilidad de uso de feromonas sintéticas en la terapia conductista ansiolítica. Entre las especies vegetales empleadas en la etnoveterinaria, se destaca Nepeta cataria, conocida popularmente como catnip. Probablemente, su efecto se deba a la acción de su principal componente, la nepetalactona sobre el sistema límbico del animal. Al realizar un análisis in chemico, se observa semejanza de este compuesto al neurotransmisor serotonina, lo que podría justificar el bienestar de los gatos, pero no hay estudios farmacológicos y toxicológicos debidamente validados. Así, se resalta la importancia de la orientación del uso racional de medicamentos y plantas medicinales, así como el uso de métodos de enriquecimiento ambiental para la reducción del estrés y ansiedad en gatos domésticos, proporcionando su mayor calidad de vida.

Palabras clave: Comportamiento felino. Ansiedad. Farmacoterapia. Etnoveterinario

\section{REFERÊNCIAS}

ABEBE, W. Herbal medication: potential for adverse interactions with analgesic drugs. Journal of Clinical Pharmacy and Therapeutics, v. 27, n. 6, p. 391-401, 2002.

\author{
ALHO, A. M. P. V. A. O enriquecimento ambiental como estratégia de tratamento e \\ prevenção da cistite idiopática felina. Lisboa: UTL, 2012. 144p. Dissertação (Mestrado \\ Integrado em Medicina Veterinária), Faculdade de Medicina Veterinária, Universidade \\ Técnica de Lisboa, 2012.
}


ALMEIDA, K. S.; FREITAS, F. L. C.; PEREIRA, T. F. C. Etnoveterinária: A Fitoterapia na Visão do Futuro Profissional Veterinário. Revista Verde de Agroecologia e Desenvolvimento Sustentável, v. 1, n. 1, p. 67-74, 2006.

ANDRADE, S. F. Manual de Terapêutica Veterinária. 2. ed. São Paulo: Roca, 2002.

AYDIN, S.; BEIS, R.; OZTÜRK, Y.; et al. Nepetalactone: a new opioid analgesic from Nepeta caesarea Boiss. Journal of Pharmacy and Pharmacology, v. 50, n. 7, p. 813-817, 1998.

BAGLEY, R. S. Anticonvulsants. In PETERSON, M. E.; TALCOTT, P. A. Small Animal Toxicology. 2. ed. Saint Louis: Saunders, 2006. P. 941-954.

BARBOSA, J. M. M. M. Uma abordagem da Fitoterapia na Medicina Veterinária. Patos: UFCG, 2011. 40p. Monografia (Graduação em Medicina Veterinária), Curso de Medicina Veterinária, Centro de Saúde e Tecnologia Rural Campus de Patos, Universidade Federal de Campina Grande, 2011. Disponível em:

<http://www.cstrold.sti.ufcg.edu.br/grad_med_vet/mono2011_1/jonia_maria_martins_mar ques_barbosa.pdf>.

BEAVER, B. V. Comportamento Felino: Um Guia para Veterinários. 2. ed. São Paulo: Roca, 2005. 384p.

BOL, S.; CASPERS, J.; BUCKINGHAM, L.; et al. Responsiveness of cats (Felidae) to silver vine (Actinidia polygama), Tatarian Honeysuckle (Lonicera tatarica), valerian (Valeriana

officinalis) and catnip (Nepeta cataria). BMC Veterinary Research, v. 13, n. 70, p. 1-15, 2017.

BRADSHAW, J. W. S. Sociality in cats: a comparative review. Journal of Veterinary Behavior, v. 11, p. 113-124, 2016.

BRADSHAW, J. W. S. Normal feline behaviour:...and why problem behaviours develop. Journal of Feline Medicine and Surgery, v. 20, n. 5, p. 411-421, 2018.

BRAGA, J. E. F.; PORDEUS, L. C.; SILVA, T. M. C.; et al. Ansiedade Patológica: Bases Neurais e Avanços na Abordagem Psicofarmacológica. Revista Brasileira de Ciências da Saúde, v. 14, n. 2, p. 92-100, 2010.

BROOM, D. M. Indicators of poor welfare. British Veterinary Journal, v. 142, n. 6, p. 524-526, 1986.

BROOM, D. M.; MOLENTO, C. F. M. Bem-estar animal: conceito e questões relacionadasrevisão. Archives of Veterinary Science, v. 9, n. 2, p. 1-11, 2004.

BRUNO, L. O.; MARQUES, L. C.; CARDOSO, C. M. Z. Análise das normas vigentes para registro de fitoterápicos veterinários no Brasil. Science and Animal Health, v. 4, n. 3, p. 209-227, 2016. 
CAMERON, M. E.; CASEY, R. A.; BRADSHAW, J. W. S.; et al. A study of environmental and behavioral factors that may be associated with feline idiopathic cystitis. Journal of Small Animal Practice, v. 45, n. 3, p. 144-147, 2004.

CAVALCANTI, M. A importância dos flavonóides naturais na Medicina Veterinária e na Terapia do Stress de animais de companhia. São Paulo: FACIS, 1997. 50p. Dissertação (Mestrado - Área de Fitoterapia), Faculdade de Ciências da Saúde de São Paulo, 1997.

CHEW, D. J.; BUfFINGTON, T. C. A.; KENDALL, M. S.; DIBARTOLA, S. P.; WOODWORTH, B. E. Amitriptyline treatment for severe recurrent idiopathic cystitis in cats. Journal of the American Veterinary Medical Association, v. 213, n. 9, p. 1282-1286, 1998.

CONFESSOR, M. V. A.; MENDONÇA, L. E. T.; MOURÃO, J. S.; et al. Animals to heal animals: ethnoveterinary practices in semiarid region, Northeastern Brazil. Journal of Ethnobiology and Ethnomedicine, v. 5, n. 37, p. 1-9, 2009.

CROWELL-DAVIS, S. L.; MURRAY, T. Veterinary Psycopharmacology. lowa: Blackwell Publishing, 2005. 270p.

CUNHA, A. P.; SILVA, A. P.; ROQUE, O. R. Plantas e Produtos vegetais em Fitoterapia. 4. ed. Lisboa: Fundação Calouste Gulbenkian, 2012.

ELLIS, S. L. H.; WELLS, D. L. The influence of olfactory stimulation on the behaviour of cats housed in a rescue shelter. Applied Animal Behaviour Science, v. 123, n. 1-2, p. 56-62, 2010.

ELLIS, S. H. L.; RODAN, I.; CARNEY, H. C. AAFP and ISFM feline environmental needs guidelines. Journal of Feline Medicine and Surgery, v. 15, p. 219-230, 2013.

ESPÍN-ITURBE, L. T.; LÓPEZ YAÑEZ, B. A.; CARRASCO GARCÍA, A.; et al. Active and passive responses to catnip (Nepeta cataria) are affected by age, sex and early gonadectomy in male and female cats. Behavioural processes, v. 142, p. 110-115, 2017.

FERREIRA, T. C.; SOUSA, C. V. S.; COSTA, P. P. C. Transtorno Obsessivo Compulsivo em cães e gatos. Revista de Ciência Veterinária e Saúde Pública, v. 3, n. 1, p. 37-43, 2016.

GAZZANO, A.; BIANCHI, L.; CAMPA, S.; MARITI, C. The prevention of undesirable behaviors in cats: Effectiveness of veterinary behaviorists' advice given to kitten owners. Journal of Veterinary Behavior, v. 10, n. 6, p. 535-542, 2015.00

GOURKOW, N.; HAMON, S. C; PHILLIPS, C. J. C. Effect of gentle stroking and vocalization on behaviour, mucosal immunity and upper respiratory disease in anxious shelter cats.

Preventive Veterinary Medicine, v. 117, n. 1, p. 266-275, 2014.

GRAEFF, F.G.; HETEM, L. A. B. Transtornos da Ansiedade. São Paulo: Atheneu, 2004. 434p. 
HART, B. L.; CLIFF, K. D.; TYNES, V. V.; et al. Control of urine marking by use of long-term treatment with fluoxetine or clomipramine in cats. Journal of the American Veterinary Medical Association, v. 226, n. 3, p. 378-382, 2005.

HATCH, R. C. Effect of drugs on catnip (Nepeta cataria) - induced pleasure behavior in cats. American Journal of Veterinary Research, v. 33, p. 143-155, 1972.

HOLM, L.; LADEWIG, J. The effect of housing rats in a stimulus rich versus stimulus poor environment on preference measured by sigmoid double demand curves. Applied Animal Behaviour Science, v. 107, n. 3-4, p. 342-354, 2007.

IKI, T.; AHRENS, F.; PASCHE, K. A.; et al. Relationships between scores of the feline temperament profile and behavioural and adrenocortical responses to a mild stressor in cats. Applied Animal Behaviour Science, v. 132, n. 1-2, p. 71-80, 2011.

JONGMAN, E. C. Adaptation of domestic cats to confinement. Journal of Veterinary Behaviour, v. 2, n. 6, p. 193-196, 2007.

KINGHORN, A. D.; PAN, L.; FLETCHER, J. N.; et al. The relevance of higher plants in lead compound discovery programs. Journal of Natural Products, v. 74, n. 6, p. 1539-1555, 2011.

KRONEN, P. W.; LUDDERS, J. W.; ERB, H. N.; et al. A synthetic fraction of feline facial pheromones calms but does not reduce struggling in cats before venous catheterization. Veterinary Anaesthesia and Analgesia, v. 33, n. 4, p. 258-265, 2006.

LAMPERT, M. Benefícios da relação homem-animal. Porto Alegre: UFRGS, 2014, 24p. Monografia (Trabalho de Conclusão de Curso), Faculdade de Veterinária, Universidade Federal do Rio Grande do Sul, 2014. Disponível em:

<https://lume.ufrgs.br/handle/10183/104881> .

LANDSBERG, G.; HUNTHAUSEN, W.; ACKERMAN, L. Problemas comportamentais do cão e do gato. São Paulo: Roca, 2004. 492p.

LEVINE, E. D. Feline fear and anxiety. Veterinary Clinics of North America: Small Animal Practice, v. 38, n. 5, p. 1065-1079, 2008.

LITTLE, S. E. The Cat: Clinical Medicine and Management. Missouri: Elsevier Saunders, 2011.

MANN, C; STABA, E. J. The chemistry, pharmacology and commercial formulations of chamomile. In: CRAKER, L. E.; SIMON, J. E. (Editors). Herbs, Spices and Medicinal Plants:

Recent Advances in Botany, Horticulture and Pharmacology. Phoenix: Oryx Press, 1986. P. 235-280. 
MASON, G.; CLUBB, R.; LATHAM, N.; VICKERY, S. Why and how should we use environmental enrichment to tackle stereotypic behaviour? Applied Animal Behaviour Science, v. 102, n. 34, p. 163-188, 2007.

MILLS, D. Pheromonatherapy: theory and applications. In Practice, v. 27, n. 7, p. 368-373, 2005.

MILLS, D.; KARAGIANNIS, C.; ZULCH, H. Stress-Its Effects on Health and Behavior: A Guide for Practitioners. Veterinary Clinics of North America: Small Animal Practice, v. 44, n. 3, p. 525-541, 2014.

MONTEIRO, M. V. B.; BEVILAQUA, C. M. L.; CAMURÇA-VASCONCELOS, A. L. F. Metodologia aplicada a levantamentos etnoveterinários. Veterinária em Foco, v. 9, n. 1, p. 1-12, 2011.

NEWMAN, D. J.; CRAGG, G. M.; SNADER, K. M. Natural products as sources of new drugs over the period 1981 - 2002. Journal of Natural Products, v. 66, n. 7, p. 1022-1037, 2003.

OLSSON, I. A. S.; NEVISON, C. M.; PATTERSON-KANE, E. G.; et al. Understanding behavior: the relevance of ethological approaches in laboratory animal Science. Applied Animal Behaviour Science, v. 81, n. 3, p. 245-264, 2003.

OMS - ORGANIZACIÓN MUNDIAL DE LA SALUD. Estrategia de la OMS sobre medicina tradicional (2014-2023). Hong Kong: Biblioteca de la OMS, 2013. 75p. Disponível em: <https://apps.who.int/iris/bitstream/handle/10665/95008/9789243506098_spa.pdf;jsession id=83480B780D9836759EC400F5C331FD45 ?sequence $=1>$.

OSTERHOUDT, K. C.; LEE, S. K.; CALLAHAN, J. M.; HENRETIG, F. M. Catnip and the alteration of human consciousness. Veterinary and Human Toxicolology, v. 39, n. 6, p. 373-375, 1997.

OVERALL, K. L. Manual of Clinical Behavioral Medicine for Dogs and Cats. St. Louis: Elsevier, 2013. 832p.

OZAKI, A. T.; DUARTE, P. C. Fitoterápicos utilizados na medicina veterinária, em cães e gatos. Infarma, v. 18, n. 11/12, p. 17-25, 2006.

PASSOS, C. S.; ARBO, M. D.; RATES, S. M. K.; et al. Terpenóides com atividade sobre o sistema Nervoso Central (SNC). Revista Brasileira de Farmacognosia, v. 19, n. 1a, p. 140-149, 2009.

PEETERS, E.; DRIESSEN, B.; STEEGMANS, R.; et al. Effect of supplemental tryptophan, vitamin $E$, and a herbal product on responses by pigs to vibration. Journal of Animal Science, v. 82, n. 8, p. 2410-2420, 2004.

PEREIRA, J. T.; PEREIRA, G. D. G. Comportamento Social dos gatos. In: FARACO, C. B.; SOARES, G. M. Fundamentos do Comportamento Canino e Felino. São Paulo: Editora MedVet, 2013. P. 145-172. 
PLUMB, D. C. Veterinary Drug Handbook. 4. ed. lowa: lowa State Press, 2002. 1436p.

PLUMB, D. C. Pharmacist's Guide to Veterinary Prescriptions: A Whole Different Animal. Plumb's Veterinary Medications Guide, 2015. Disponível em:

$<$ https://www.plumbsveterinarydrugs.com/files/pharmacists-guide/pharmacists-guidev3.pdf> .

POPPENGA, R. H. Herbal medicine: potential for intoxication and interactions with conventional drugs. Clinical Techniques in Small Animal Practice, v. 17, n. 1, p. 06-18, 2002

SCOTT, D. W.; MILLER, H. W.; GRIFFIN, C. E. Muller and Kirk's Small Animal Dermatology. 6. ed. Philadelphia: Saunders, 2001.

SHERRY, C. J.; HUNTER, P. S. The effect of an ethanol extract of catnip (Nepeta cataria) on the behaviour of the Young chick. Experientia, v. 35, n. 2, p. 237-238, 1979.

SILVA, B. P. L.; KNACKFUSS, F. B.; LABARTHE, N.; et al. Effect of a synthetic analogue of the feline facial pheromone on salivary cortisol levels in the domestic cat. Pesquisa Veterinária Brasileira, v. 37, n. 3, p. 287-290, 2017.

SIMPSON, B. S.; PAPICH, M. G. Pharmacologic management in veterinary behavioral medicine. Veterinary Clinics of North America: Small Animal Practice, v. 33, n. 2, p. 365404, 2003.

SPINOSA, H. S.; GÓRNIAK, S. L.; PALERMO-NETO, H. S. Toxicologia Aplicada a Medicina Veterinária. São Paulo: Manole, p. 117-133, 2008.

STELLA, J.; CRONEY, C.; BUFFINGTON, T. Effects of stressors on the behavior and physiology of domestic cats. Applied Animal Behaviour Science, v. 143, p. 157-163, 2013.

TODD, N. B. The catnip response. Cambridge: HU, 1963. 61p. Thesis (Doctor of Philosophy in the subject of Biology), Department of Biology, Harvard University, 1963. Disponível em: $<$ https://www.gwern.net/docs/catnip/1963-todd.pdf>.

USDA - U. S. DEPARTMENT OF AGRICULTURE. Agricultural Research Service. Germplasm Resources Information Network (GRIN-Taxonomy). National Plant Germplasm System, 2018. Disponível em: <https://www.ars-grin.gov/> .

VÉGAS, C. Fitoterapia é nova medicina animal. Tribuna, 2007. Disponível em: https://www.tribunapr.com.br/noticias/mundo/fitoterapia-e-nova-medicina-animal/

VILANOVA, X. M. Etología Clínica Veterinaria del Perro y del Gato. 3. ed. Barcelona: Multimédica Ediciones Veterinarias, 2003. 
WAISGLASS, S. E.; LANDSBERG, G. M.; YAGER, J. A.; HALL, J. A. Underlying medical conditions in cats with presumptive psychogenic alopecia. Journal of the American Veterinary Medical Association, v. 228, n. 11, p. 1705-1709, 2006.

ZHU, J. J.; ZENG, X. P.; BERKEBILE, D.; et al. Efficacy and safety of catnip (Nepeta cataria) as a novel filth fly repelente. Medical and Veterinary Entomology, v. 23, n. 3, p. 209-216, 2009.

Autora para correspondência: Professora Doutora Edna Sayuri Suyenaga. Av. Unisinos 950, CEP 93022-000, São Leopoldo, Brasil. ednafarm@yahoo.com.br 